\title{
Caries Temprana de la Infancia en la Ciudad Autónoma de Buenos Aires. Comparación de frecuencia en diferentes ámbitos de atención
}

Ana María Biondi ${ }^{1}$, Silvina Gabriela Cortese 2; Andrea Ortolani ${ }^{3}$

\section{Resumen}

Objetivos: Comparar la edad de primera consulta y la frecuencia de caries temprana de la infancia entre los pacientes que demandan atención en un servicio público arancelado y los que lo hacen en consultorios odontopediátricos privados en la misma zona geográfica. Material y Métodos: Diseño observacional retrospectivo. Fueron evaluadas todas las Historias Clínicas de los pacientes que concurrieron por primera vez a la Cátedra Odontología Integral Niños (G1) y a 2 consultorios privados (G2) durante el periodo enero-diciembre 2015. (Kappa > 0.83). De los menores de 6 años, los registros de edad de primera consulta, sexo y ceos fueron ingresados a una base de datos Excel para la obtención de los resultados expresados en porcentajes, medias y desvío estándar. Las comparaciones se realizaron utilizando ANOVA y Chi cuadrado. Resultados: La mues- tra total se conformó con 973 niños en G1 y 488 en $\mathrm{G} 2$, de $9,3 \pm 3,8$ y $4,9 \pm 3$ años respectivamente $(\mathrm{p}=0,000)$. Los menores de 6 años representaron el 16\% (G1m) y 63,5\% (G2m) ( $p=0,000)$. La edad fue de $51 \pm 14$ y $39,5 \pm 13$ meses ( $p=0,000)$; masculinos: $54,8 \%$ y $52,9 \%(p=0,41)$, ceos $=17,4 \pm 15,4$ $(12,5 \%$ ceos $=0) y=0,45 \pm 1,6 \quad(85,8 \%$ ceos $=0)$ $(p=0.000)$ y la frecuencia de caries temprana de la infancia fue de $87,5 \%$ (IC 79,5-93,2) y 2,26\% (IC $0,90-4,60)(p=0,000)$ para G1m y G2m respectivamente. Conclusiones: En el servicio público arancelado la consulta se realiza mayormente en forma tardía y los que la realizan a edades tempranas presentan severos índices de patología. Los pacientes con cobertura de salud demandan atención a edades tempranas con criterio preventivo.

Palabras Clave: Caries dental, niño, prevalencia, Primera consulta odontológica, Caries Temprana de la Infancia.

\footnotetext{
1. Profesora Titular, Cátedra Odontología Integral Niños, Facultad de Odontología, Universidad de Buenos Aires, Buenos Aires, Argentina.

2. Profesora Asociada, Cátedra Odontología Integral Niños, Facultad de Odontología, Universidad de Buenos Aires, Buenos Aires, Argentina.

3. Profesora Adjunta, Cátedra Odontología Integral Niños, Facultad de Odontología, Universidad de Buenos Aires, Buenos Aires, Argentina.
} 
Artigo Original

\section{Cárie Precoce na Infancia. Comparação de frequência em diferentes âmbitos de atendimento.}

\section{Resumo}

Objetivos: Comparar a idade da primeira consulta e a frequência de cárie precoce na infancia entre os pacientes que necessitam atenção em um serviço público financiado e os que o fazem em consultórios particulares (odontopediátricos) na mesma zona geográfica. Material e Métodos: Desenho observacional retrospectivo. Foram avaliadas todas as histórias clínicas dos pacientes que participaram pela primeira vez na Disciplina de Odontologia Integral de crianças (G1) e em 2 consultórios particulares (G2) durante o período de janeiro a dezembro de 2015 (Kappa >0.83). Dos menores de 6 anos, os registros de idade de primeira consulta, sexo e ceos foram introduzidos em uma base de dados Excel para a obtenção dos resultados expressos em porcentagens, medias e desvio estandar. As comparações se realizaram utilizando ANOVA e quiquadrado. Resultados: A amostra total foi composta de 973

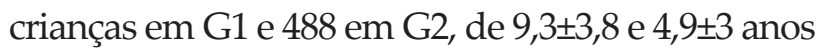
respectivamente $(\mathrm{p}<0,001)$. Os menores de 6 anos representaram 16\% (G1m) e 63,5\% (G2m) (p<0,001). Para G1 e G2, respectivamente, a idade foi de $51 \pm 14$ e 39,5 \pm 13 meses ( $p<0,001$ ); sexo masculino: $54,8 \%$ e $52,9 \%(\mathrm{p}=0,41)$, ceos: $17,4 \pm 15,4(12,5 \%$ ceos $=0)$ e $=$ $0,45 \pm 1,6(85,8 \%$ ceos $=0)(p<0,001)$ e a frequência de cárie precoce na infância foi de 87,5\% (IC 79,5-93,2) e $2,26 \%$ (IC $0,90-4,60)(p<0,001)$ para G1m y G2m respectivamente. Conclusões: No serviço público financiado a consulta se realiza, na maioria das vezes, de forma tardia e os que realizam em idade precoce apresentam índices severos da doença. Os pacientes com cobertura de saúde demandam atenção em menor idade com criterios preventivos.

Palavras chave: Cárie Dentária, Criança, Prevalência, Primeira consulta odontológica, Cárie precoce na infancia.

Original article

\section{Early Childhood Caries in the City of Buenos Aires. Comparison of frequency in different dental-care settings}

\begin{abstract}
Objectives: To compare age of first consultation and frequency of early childhood caries in patients seeking care at a public pediatric dental-care service providing care for a fee, and at private pediatric dental offices in the same geographical area. Methods: A retrospective
\end{abstract}

descriptive study design was used. All patient records of patients seeking care at the Department of Dentistry for Children (G1) and at 2 private pediatric dental offices (G2) for the first time between January and December 2015 were evaluated. $($ Kappa $=0.83)$. Records of children aged less than 6 years were reviewed and the following variables were recorded on an Ex- 
cel database: age at first consultation, sex, and dmfs. Results were expressed as a percentage, mean and standard deviation. ANOVA and Chi square test were used to compare the data. Results: The total sample comprised 973 children in G1 and 488 in G2, average age $9.3 \pm 3.8$ and $4.9 \pm 3$ years respectively $(p=0.000)$. 16\% $(\mathrm{G} 1 \mathrm{~m})$ and $63.5 \%(\mathrm{G} 2 \mathrm{~m})$ were children under 6 years old $(p=0,000)$. Average age was $51 \pm 14$ and $39.5 \pm 13$ months $(p=0,000)$, with boys accounting for $54.8 \%$ and $52.9 \%(p=0.41)$; dmfs: $=17.4 \pm 15.4$ $(12.5 \%$ were caries free) and $0.45 \pm 1.6(85.8 \%$

\section{Introducción}

La Asociación Dental Americana ${ }^{1}$ (2000), el Centro de Prevención y Control de Enfermedades de los EEUU ${ }^{2}$ (2011) y la Asociación Americana de Odontología Pediátrica ${ }^{3}$ (2014) consideran a la caries temprana de la infancia (CTI) como un problema de Salud Pública y la definen como la presencia de una o más piezas primarias con lesiones de caries cavitadas o no, perdidas o restauradas por caries en niños menores de 6 años (hasta 71 meses). En los menores de 3, la presencia de cualquier signo de caries en superficies libres es indicativa de caries severa de la infancia (CTI-S). Su etiología está asociada a determinantes biológicos, sociales y de comportamiento. La falta de implementación de medidas preventivas y de atención temprana ocasiona consecuencias tales como alto riesgo para nuevas lesiones tanto en dentición primaria como permanente, infecciones agudas en algunos casos con hospitalización, problemas estéticos, dificultades para la alimentación; además de repercusiones médicas, emocionales y económicas. ${ }^{4}$ Numerosos autores concluyeron que existe una asociación negativa entre caries y calidad de vida, debi- $\mathrm{dmfs}=0)(\mathrm{p}=0.000)$, and frequency of early childhood caries $87.5 \%$ (CI 79.5-93.2) and 2.26\% (CI $0.90-4.60)(p=0.000)$ in G1m and G2m respectively. Conclusions: As observed at the public service providing dental care for a fee, patients delay in seeking care and their index scores show severe disease at an early age. Private sector patients with health insurance coverage seek care at an early age as a preventive measure.

Keywords: Dental caries, child, prevalence, First dental visit, Early Childhood caries.

do a que los preescolares con dolor dentario no siempre se quejan, aunque lo manifiestan indirectamente cambiando los hábitos alimenticios y alterando sus patrones de sueño. 5, 6

Su prevalencia es variable en función a las características socioculturales de las poblaciones bajo estudio siendo la etiología, un tema controvertido por los múltiples factores de riesgo asociados a la misma. Los resultados de un estudio reciente realizado en Medellín, Colombia revelan que la condición de salud bucal es mejor en niños pertenecientes a estratos medios y altos en comparación con aquellos que pertenecen a estratos bajos. ${ }^{7}$ La literatura reporta valores de prevalencia de hasta el $90 \%$ en poblaciones vulnerables y del 1 al $12 \%$ en países industrializados; rangos muy diferentes donde se sugiere a la situación socioeconómica como un factor condicionante. ${ }^{8}$ La gran disparidad de resultados se atribuye en parte a las diferentes definiciones que se utilizan, características demográficas, diseños y años en que se realizaron los trabajos. ${ }^{9}$ A nivel de Salud Pública la información en Argentina es escasa. En Buenos Aires se presentó recientemente un trabajo realizado en Berisso, La Plata, en niños de riesgo 


\begin{tabular}{|l|c|c|c|}
\hline & G1m & G2 $\mathbf{m}$ & $\mathbf{p}=$ \\
\hline ceos & $17,4+15,4$ & $0,45+1,6$ & 0.000 \\
\hline ceos $=0$ & $12,5 \%$ & $85,8 \%$ & \\
\hline CTI & $\begin{array}{c}87,5 \% \\
(79,5-93,2)\end{array}$ & $\begin{array}{c}14,2 \% \\
(10,6-18,7)\end{array}$ & 0,000 \\
\hline
\end{tabular}

Tabla 1. Frecuencia de CTI y ceos en niños menores de 6 años en ambos ámbitos de atención.

social donde se reportó un índice ceod $=2.3$ en menores de 30 meses. ${ }^{10}$ En la Ciudad Autónoma de Buenos Aires los resultados de Piovano y col. del $2008,{ }^{11}$ revelaron un $22.4 \%$ de niños libres de caries a los 5 años y un ceod promedio de 5.1

En la Cátedra de Odontología Integral Niños los alumnos de pre y posgrado, y los docentes con funciones asistenciales, brindan atención integral a niños desde el nacimiento a los 16 años, que en su mayoría provienen de la Ciudad Autónoma de Buenos Aires (CABA) y del conurbano bonaerense.

En este trabajo la hipótesis planteada fue que en niños provenientes de familias con escasos recursos económicos y sin cobertura de salud la consulta se realiza en forma tardía y con severos índices de patología.

\begin{tabular}{|c|c|c|}
\hline & G1 & G2 $\mathbf{m}$ \\
\hline $\mathbf{1}$ & $\mathbf{7}$ & 2 \\
\hline $\mathbf{2}$ & $15,77+16,13$ & $0+1,1$ \\
\hline $\mathbf{3}$ & $15,68+12,89$ & $2,5+1,6$ \\
\hline $\mathbf{4}$ & $22,03+16,42$ & $0+1,3$ \\
\hline $\mathbf{5}$ & $21,12+14,02$ & $2,7+3$ \\
\hline
\end{tabular}

Tabla 2. Valores de ceos por edad en ambos grupos.
Los objetivos de este estudio fueron 1) comparar la frecuencia de caries temprana de la infancia (CTI) entre los pacientes que demandan atención en un servicio público arancelado y los que lo hacen en consultorios odontopediátricos privados en la misma zona geográfica, 2) comparar la edad de primera consulta entre ambos grupos de pacientes

\section{Métodos:}

Se diseñó un proyecto observacional retrospectivo, presentado a la Secretaria de CyT de FOUBA y aprobado por el Comité de Ética de la institución (№ 002/2017 CETICAFOUBA)

El Grupo G1 quedó conformado por todos los pacientes ingresados a la cátedra de primera consulta para atención integral, entre enero y diciembre de 2015. Se excluyeron los recitados o quienes iniciaron tratamientos en años anteriores. Los datos fueron obtenidos del libro de recepción y de las historias clínicas (HC). De las HC de los menores de 6 años que realizaron tratamiento integral se registró el índice ceos, el sexo y la edad en meses. El Grupo G2 quedó conformado por pacientes de primera consulta en consultorios odontopediátricos privados de Recoleta, CABA, donde se obtuvieron los mismos datos durante el mismo periodo. Luego que el adulto responsable firmara el consentimiento, el examen clínico fue realizado por 9 docentes estandarizados (Kappa $=0,83$ ). Los registros de las diferentes variables de ambos grupos se ingresaron a una base de datos Excel 2013 para la obtención y comparación de los resultados. Los resultados se expresaron en porcentajes, medias y desvíos estándar y se realizaron comparaciones utilizando ANOVA y Chi cuadrado. 


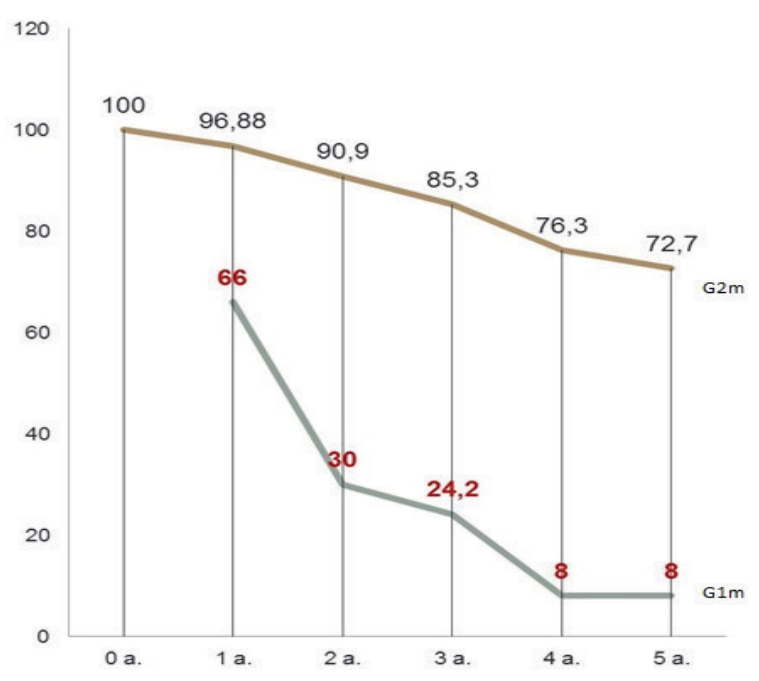

Gráfico 1. Porcentajes de niños libres de caries distribuidos por edad en los pacientes menores de 6 años en ambos grupos.

\section{Resultados:}

La muestra total se conformó con 973 niños en

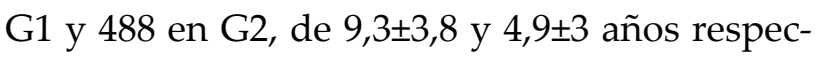
tivamente. $(p=0,000)$. La moda de la edad en G1 fue de 8 años y en G2 3 años.

Se definieron dos subgrupos G1m y G2m conformados por los pacientes menores de 6 años (hasta 71 meses) de cada uno de los grupos G1 y G2 respectivamente.

Los menores de 6 años fueron: 16\% (156 pacientes: G1m) en G1 y 64\% (312: G2m) en G2. En G1m fueron excluidas 52 historias cínicas cuyos datos no estaban completos y en G2m 12, por lo cual los subgrupos para la obtención y comparación de los resultados quedaron conformados por 104 niños en G1m y 300 en G2m.

En G1m y G2m, los porcentajes de pacientes masculinos fueron $54,8 \%$ y $52,9 \%(p=0,41)$ y las medias de edades $51 \pm 14$ meses y $39,5 \pm 13$ meses $(p=0,000)$ respectivamente. En la Tabla 1 se expresa la frecuencia de CTI y el ceos en G1m y G2m.

El gráfico 1 revela los porcentajes de niños libres de caries distribuidos por edad en los pacientes menores de 6 años en ambos grupos.

Los gráficos 2a y $2 \mathrm{~b}$ representan el número de niños por edad libres de caries y con CTI en cada grupo. .

En la Tabla 2 se expresan los valores de ceos de los pacientes menores de 6 años por edad en ambos grupos.

\section{Discusión:}

A pesar que la caries es prevenible, permanece como la enfermedad humana no contagiosa más común en la infancia. Durante esta etapa, las piezas primarias están menos mineralizadas, la saliva tiene menor concentración de Calcio, el pH crítico es significativamente mayor que en piezas permanentes de adultos por lo que las lesiones avanzan con mayor velocidad. ${ }^{12}$ La denominación de CTI reemplaza a las anteriores como Caries de Lactancia, Síndrome del Biberón y Caries Rampante, integrando en su compleja etiología factores tales como educación de los padres o cuidadores, hábitos alimenticios (lactancia materna prolongada a demanda nocturna, frecuencia en uso de mamaderas, consumo frecuente de bebidas o alimentos azucarados), defectos estructurales del esmalte y grupos con bajo nivel socio económico, entre otros. Los hijos de madres con estado de salud bucal inadecuado y alto recuento de microorganismos patógenos tienen 5 veces más posibilidades de desarrollar caries que aquellos cuyas madres presentan buen estado de salud. ${ }^{13}$ 
120

100

80

60

40

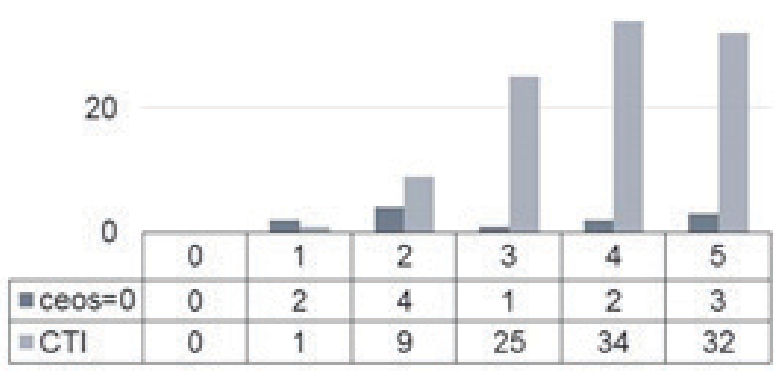

120

100

80

60

20

0

\begin{tabular}{|c|c|c|c|c|c|c|}
\hline 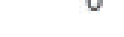 & 0 & 1 & 2 & 3 & 4 & 5 \\
\hline - ceos $=0$ & 2 & 32 & 78 & 108 & 55 & 31 \\
\hline$=\mathrm{CTI}$ & 0 & 1 & 4 & 16 & 13 & 10 \\
\hline
\end{tabular}

Gráficos $2 a$ y $2 \boldsymbol{b}$. Demandas de atención en niños con y sin caries distribuidas por edad en los pacientes menores de 6 años en ambos grupos..

El índice empleado en este trabajo, ceos (adoptado por Gruebbel, 1944) para la dentición primaria, fue utilizado porque por su simplicidad es empleado en la cátedra y en numerosos trabajos epidemiológicos en la literatura nacional e internacional, aunque al no registrar las lesiones en la fase de precavitación, conduce a una subvaloración importante de la prevalencia real de la enfermedad.

Con el término Caries Temprana de la Infancia Severa (CTI-S) se define a cualquier signo de caries, con o sin cavitación, en niños menores de 3 años y con el de Caries Severa de la Infancia asociada a Hipoplasia (HAS-ECC por su sigla en inglés) a la CTI-S en niños con hipoplasia como consecuencia de una estructura hipomineralizada que representa el momento del nacimiento que se hace más acentuada por la presencia de uno o varios factores durante período prenatal o infancia temprana como partos laboriosos, asfixia e infección neonatal, bebés prematuros, sarampión, madres diabéticas, madres y/o bebés desnutridos, entre otros. En estos niños, una dieta alta en hidratos de carbono y baja en proteínas y una temprana colonización por bacterias cariogénicas aumentan hasta 15 veces las probabilidades de desarrollar caries en niños comparados con otros. ${ }^{14}$ En este trabajo no se indagó sobre antecedentes del embarazo ni del nacimiento, así como tampoco sobre características de la dieta, identificando solo valores de patología en niños menores de 3 años que corresponderían a CTI-S.

Los resultados de Squassi y col (2008) en Argentina, ${ }^{15}$ Baldani y col (2011) en Brasil,$^{16}$ y Zhou y col. (2011) en China, ${ }^{17}$ entre otros, analizaron variables vinculadas con la pobreza y su relación con la salud bucodental y concluyeron que los grupos vulnerables presentaron 
mayor riesgo y actividad cariogénica. Baggio y col $(2015)^{18}$ sugirieron considerar a la CTI como un indicador de desigualdad social. Este estudio reveló resultados muy diferentes en relación a la frecuencia de CTI e índice ceos en dos poblaciones de semejante edad y sexo demandando atención en dos ámbitos diferentes. La población que concurre a la cátedra se constituye en su mayoría de niños provenientes de familias con escasos recursos económicos $\mathrm{y}$ sin cobertura de salud. La frecuencia de CTI en el grupo de niños < a 6 años fue de 87,5\%, semejante al $90 \%$ observado en comunidades aborígenes en Canadá, ${ }^{19}$ al $89.2 \%$ en Kosovo, ${ }^{20}$ y al $88,6 \%$ registrado en instituciones educativas de estratos bajos en Medellín, Colombia a los 5 años ${ }^{7}$ y mayor al 36\% observado en un estudio sobre 369 niños de bajo nivel sociocultural en Brasil. ${ }^{21}$ Si bien en todos los estudios se trata de poblaciones con diferentes estilos de vida que pueden constituirse como factores de riesgo, todas ellas se encontraban en situación de vulnerabilidad social, teniendo en cuenta para este trabajo factores asociados a ella tales como nivel educativo alcanzado por los padres y cobertura de salud efectiva, registrados ambos en la historia clínica. La media del ceos de $2.5 \pm 1.6$ a los 3 años hallada en consultorios privados con pacientes provenientes mayormente de familias de perfil socioeconómico de alto nivel educativo y niveles de ingresos medios a medio-altos fue semejante a la hallada por Davies y col $(2001)^{22}$ de $2.8 \pm 7$ en tres distritos de Manchester, UK. Con respecto a los niños que a esta edad presentaron un ceos $>0$ fue similar al obtenido por Nordblad y Col. ${ }^{23}$ en 2004 en Finlandia.

En ambos grupos se trata de niños que concurren con demanda de atención por lo que los valores obtenidos son más altos que en poblaciones sin demanda, enfatizando que la patología de los pacientes que concurren a la cátedra es severa teniendo en cuenta que se trata de un centro de referencia, situación que también se presentó en los consultorios privados por tratarse de especialistas con alto nivel de derivación. La Cátedra de Odontología Integral Niños cumple tareas de docencia, investigación y asistencia que promueven las consultas precoces a través de los contenidos de la asignatura, de tareas de Educación para la Salud con los acompañantes de los pacientes y con una Clínica del Bebé. Por otra parte, la Facultad de Odontología de la Universidad de Buenos Aires implementa programas preventivos y asistenciales desde la Secretaría de Extensión y la Cátedra de Odontología Preventiva y Comunitaria en poblaciones vulnerables. También los hospitales públicos brindan asistencia gratuita, pero todas estas acciones son insuficientes si no se articulan con políticas públicas con énfasis en acciones tempranas.

Los resultados de este trabajo reflejan un perfil inverso de los pacientes que demandan atención en consultorios privados por primera vez, que lo hacen mayoritariamente a los 3 años en estado de salud, en relación a los que demandan atención en el servicio público arancelado, que la realizan mucho más tardíamente, con frecuencia a los 8 años y quienes lo hacen a menor edad presentan índices de patología dentaria mucho mayores. Esta situación ocasiona tratamientos largos, con mayores costos, requerimientos de prácticas invasivas de difícil aceptación para niños de corta edad y necesidad de retratamientos. Recientes resultados presentados por el Observatorio de la Deuda Social Argentina (ODSA-UCA Año 2010-2015) ${ }^{24}$ expresan que el $48 \%$ de los niños 
en 2015 carecían de cobertura de salud, y 47\% presentaban déficit de control odontológico en la Argentina urbana. Esta situación deja expuesto un problema social relacionado con la educación sobre hábitos de alimentación e higiene y valoración de la salud de las piezas primarias comprometiendo el futuro de la dentición permanente.

\section{Conclusiones:}

En el servicio público arancelado la consulta se realiza mayormente en forma tardía y los que la realizan a edades tempranas presentan severos índices de patología. Los pacientes con cobertura de salud demandan atención a edades tempranas con criterio preventivo.

\section{Referencias bibliográficas}

1. American Dental Association. Statement on Early Childhood Caries. 2000. Disponible en: http://www.ada.org/en/about-theada/ada-positions-policies-and-statements/statement-on-early-childhood-caries.

2. Centers for Disease Control and Prevention (CDC) (2011) Oral Health: preventing cavities, gum disease, tooth loss, and oral cancers. At a Glance 2011. http://www.cdc.gov/chronicdisease/resources/publications/AAG/doh.htm.

3. American Academy of Pediatric Dentistry. Policy on Early Childhood Caries (ECC): Classifications, Consequences, and Preventive Strategies. Revisado 2014. Disponible en: http://www.aapd.org/media/policies_guidelines/p_eccclassifications.pdf.

4. Villena Sarmiento R, Pachas Barrionuevo F, Sánchez Huaman Y, Carrasco Loyola M. Prevalencia de caries de infancia temprana en niños menores de 6 años de edad residentes en poblados urbano marginales de Lima Norte. Rev Estomatol Herediana 2011; 21(2): 79-86.

5. Echeverría López S, D Aquino E, Sepúlveda Ramírez, Barra Pérez M. Cares temprana de la infancia severa: Impacto en la Calidad de Vida relacionada a la Salud Oral de Niños preescolares. Rev. Dent. Chil. 2010; 101 (2) 15-21.

6. Filstrup S, Briskie D, Fonseca M, Lawrence L, Wandera A, Inglehart M. Early childhood caries and quality of life: child and parent perspectives. Pediatr. Dent. 2003; 25(5): 431-440.

7. Blanca S. Ramírez-Puerta; Angela M. Franco-Cortés ; Emilia Ochoa-Acosta ; Gloria Escobar-Paucar Experiencia de caries en dentición primaria en niños de 5 años, Medellín, Colombia http://www.scielo.org.co/pdf/rfnsp/v33n3/v33n3a03.pdf.

8. Prakash P, Subramaniam P, Durgesh BH, Konde S. Prevalence of early childhood caries and associated risk factors in preschool children of urban Bangalore, India: A cross-sectional study. European Journal of Dentistry. 2012; 6(2): 141-152. 9. Ismail AI, Sohn W. A systematic review of clinical diagnostic criteria of Early Childhood Caries. J Public Health Dent. 1999; 59: 171-191.

10. Ruiz, M E. Estudio clínico epidemiológico de prevalencia de caries en niños de 0 a 30 meses y determinación de los factores de riesgo en una población de la ciudad de Berisso (Tesis Doctoral). Repositorio institucional de la UNLP. 2015. Disponible en: http://sedici.unip.edu.ar/handie/10915/52493.

11. Piovano S, Bordoni N, Doño R, Argentieri A, Cohen A, Klemonskis G, Macucho ME, Pedemonte Z, Pistochini A, Squassi

A. Estado dentario en niños, adolescentes y adultos de la ciudad autónoma de Buenos Aies. Revista de la Facultad de Odontologia.(UBA) 2008, 23 (54/55): 34-42.

12. Anderson $\mathrm{P}$, Hector MP, Rampersad MA. Critical $\mathrm{pH}$ in resting and stimulated whole saliva in groups of children and adults. Int J Paediatr Dent. 2001 Jul; 11(4) :266-73. 
13. Brown JP, Dodds MWJ - Dental Caries and Associated Risk Factors Capítulo 4 en: Prevention in Clinical Oral Health Care. 7a ed. St. Louis, Mo: Mosby Elsevier, 2008.

14. Caufield PW, Li Y, Bromage TG. Hypoplasia-associated severe early childhood caries-a proposed definition. J Dent Res. 2012; 91(6): 544-50.

15. C. Squassi A, Mauro S, Mauro MJ, Sánchez G, Bordoni N. Relation between oral health in children and poverty related factors. Acta Odontol Latinoam. 2008.

16. D. Badani MH, Mendes YBE, Lawder JAC, de Lara API, Rodrigues MMAS, Antunes JLF. Inequalities in dental services utilization among Brazilian low-income children: the role of individual determinants. J Public Health Dent. 2011.

17. Zhou Y, Lin H, Lo E, Wong M. Risk indicators for early childhood caries in 2-year-old children in southern China. Aust Dent J. 2011.

18. Baggio S, Abarca M, Bodenmann P, Gehri M, Madrid C. Early childhood caries in Switzerland: a marker of social inequalities. BMC Oral Health. 2015.

19. Irvine JD, Holve S, Krol D, Schroth R. Early childhood caries in Indigenous Canadian communities. Paediatric Society , First Nations, Inuit and Métis Health Committee Paediatr Child Health 2011; 16(6): 351-7.

20. Shabani LF, Begzati A, Dragidella F, Hoxha VH, Cakolli VH, et al. The Correlation between DMFT and OHI-S Index among 10-15 Years Old Children in Kosova. Journal of Dental and Oral Health, 2015 Disponible en http://scientonline.org/ open-access/the-correlation-between-dmft-and-ohi-s-index-among-10-15-years-old-children-in-kosova.pdf.

21. Azevedo TD, Bezerra AC, de Toledo OA. Feeding habits and severe early childhood caries in Brazilian preschool children. Pediatr Dent 2005; 27: 28-33.

22. Davies GM, Blinkhorn FA, Duxbury JT. Caries among 3-year-olds in greater Manchester. Br Dent J. 2001; 190: $381-4$.

23. H. Nordblad A, Souminen-Taipale L, Rasilainen J, Karhunen T. Suun terveydenhuoltoa terveyskeskuksissa 1970luvulta vuoteen 2000 (Oral Health Care at Health Centers from the 1970s to the year 2000). Helsinki: National Research and Development Center for Welfare and Health (STAKES), Report 278, 2004.

24. ODSA-UCA. Pobreza y desigualdad por ingresos en la argentina urbana 2010-2015. Tiempos de balance Disponible en http:// www.uca.edu.ar/uca/common/grupo68/files/2016-Obs-Informe-n1-Pobreza-Desigualdad-Ingresos-Argentina-Urbana.pdf.

Recibido: 26/01/2017

Aceptado 22/05/2017

Correspondencia: Ana María Biondi anamariabiondi@hotmail.com M T de Alvear 2142.

Piso 15. C1122AAH CABA. Argentina TE: +54115287-6434 